occurred every day but at erratic times, so that the mothers could not comfort their pups (termed the F1 generation) with extra cuddling before separation.

When raised this way, male offspring showed depressive behaviours and tended to underestimate risk, the study found. Their sperm also showed abnormally high expression of five microRNAs. One of these, miR-375, has been linked to stress and regulation of metabolism.

The F1 males' offspring, the F2 generation, showed similar depressive behaviours, as well as abnormal sugar metabolism. The $\mathrm{F} 1$ and $\mathrm{F} 2$ generations also had abnormal levels of the five microRNAs in their blood and in the hippocampus, a brain region involved in stress responses. Behavioural effects persisted in the F3 generation as well.

Mansuy and her team are now looking into whether similar microRNA biomarkers occur in people exposed to traumatic events - or in their children. "If some are altered persistently in blood, then they could be used as markers for susceptibility to stress or for developing psychiatric disorders," she says.

To rule out the possibility that the effects of stress were transmitted socially, the researchers also collected RNA from the F1 males' sperm and injected it into freshly fertilized eggs from untraumatized mice. This resulted in mice with comparable depressive behaviours and metabolic symptoms - and the depressive behaviours were passed, in turn, to the next generation.

The authors readily admit that there is much to be discovered about the biological underpinnings of these findings. No one knows how stress

triggers the changes in sperm microRNA, for example. One potential route is through glucocorticoid receptors, proteins involved in the stress response that are expressed

"Dadis having a much larger role... than just delivering his genome." in sperm. It could be that stress hormones circulating in the blood make their way to the testes and bind to these receptors, somehow triggering changes in microRNA expression, says Sarah Kimmins, an epigeneticist at McGill University in Montreal, Canada. "Nobody's explored that, and I think that's a really exciting avenue."

Krawetz notes another puzzle raised by the study - the stressful experience did not affect the sperm microRNA of the F2 or F3 generations. This could mean that the abnormalities in these progeny came from some other epigenetic mechanism, such as DNA methylation or chemical marks on histones, the proteins that DNA wraps around. But for now, he says, "that's all hand-waving". -

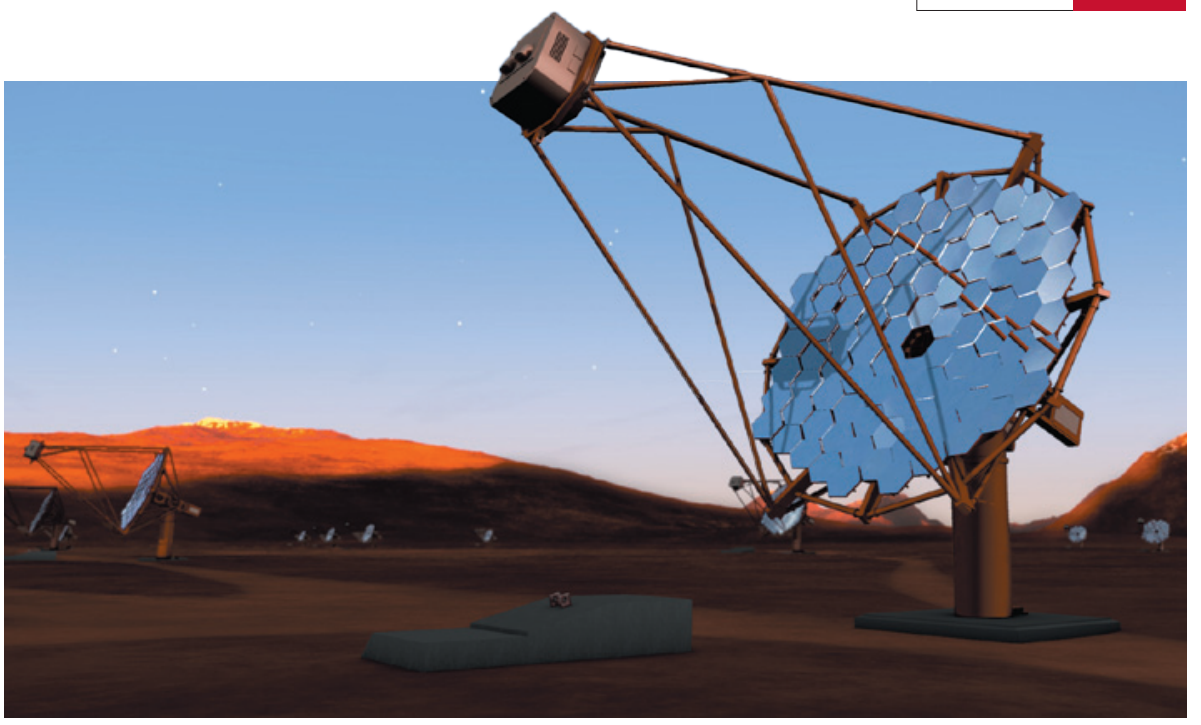

The telescope array (artist's impression) will be split across the Northern and Southern hemispheres.

ASTRONOMY

\title{
Panel homes in on sites for $\gamma$-ray detector
}

\section{Cherenkov Telescope Array will track high-energy photons to probe black holes, dark matter and relativity.}

\section{BY ELIZABETH GIBNEY}

$\mathrm{W}$ hen very-high-energy $\gamma$-rays slam into Earth's atmosphere, they trigger particle showers that emit a faint blue light. With this light, astronomers want to trace the rare $\gamma$-rays - only a few strike each square metre of the atmosphere each month - back to their sources, violent objects such as supermassive black holes. But first researchers must find a home for the planned $€ 200$-million (US\$277-million) Cherenkov Telescope Array (CTA) - or rather, two homes. The telescope will be made up of a 19-dish array in the Northern Hemisphere and a 99-dish array in the south.

At a meeting in Munich, Germany, on 10 April, representatives from the 12 CTA partner countries inched closer to picking the sites. In the Southern Hemisphere, they narrowed the list down to two possibilities: Aar, in southern Namibia; and Cerro Armazones in Chile's Atacama Desert. In the north, four sites remain in the running: two in the United States and one each in Mexico and Spain.

Some had hoped the panel would pick firm favourites. Last year, a committee of CTA scientists came up with a broader list of sites based on environmental factors such as weather and earthquake risk. The latest decision adds considerations such as political stability and the financial contributions of host nations. "The process is going slower than we'd like, but it's going, and that's great," says Rene Ong, a physicist at the University of California, Los Angeles, who has helped to plan for the CTA.

The array would study photons in an as-yet unexplored energy region: up to 100 teraelectronvolts. Cosmic rays - protons and other nuclei - emit these photons when they are accelerated at the surface of neutron stars and black holes, and when they collide in stellar winds.

The CTA would focus on the centre of the Milky Way because of the dark matter thought to lurk there; many theories predict that darkmatter particles could annihilate each other and emit $\gamma$-rays that the CTA should detect. The array would explore physics at energy scales well beyond the scope of most powerful accelerators.

The CTA would also probe theories of quantum gravity, which try to reconcile quantum mechanics with Einstein's theory of gravity. Some theories predict that very-high-energy photons, with wavelengths approaching the foamy quantum scale of space-time, will travel slightly slower than lower-energy photons from the same source. Observations of $\gamma$-rays at different energies could reveal arrival-time lags.

The CTA panel aims to pick a final southern site by the end of the year. Choosing the northern site may take longer, says panel chair Beatrix Vierkorn-Rudolph, deputy directorgeneral of Germany's Federal Ministry of Education and Research. Astronomers hope to be ready to start construction by the end of 2015 and to begin full operations in around 2020. 